\title{
Synthon evolution in trifluoromethyl anilines: In- situ cryocrystallographic study
}

Sathishkumar Ranganathan ${ }^{1}$, Munirathinam Nethaji ${ }^{1}$

${ }^{1}$ Inorganic And Physical Chemistry, Indian Insitute Of Science, Bangalore, India E-mail: sashe003@gmail.com

Anilines in general and fluorinated anilines in particular are used as precursors in the synthesis of various organic compounds of biological and industrial importance. As they form an integral part of many important molecules, the knowledge of intermolecular interactions in their ensuing crystal structures will provide deeper insights towards the design of new functional materials. Also, these compounds provide a unique system for studying the role of weak intermolecular interactions, especially those involving fluorine, in guiding the overall solid state structures[1]. Most of the anilines are liquids at ambient conditions abandoning the study of their crystal structures under normal conditions. In this respect, in-situ cryocrystallography technique has been successfully employed for the determination of the crystal structures of three isomeric compounds [2], namely ortho, meta and para-trifluoromethyl anilines which are liquids at room temperature. The crystal structures have been analyzed for various intermolecular $\mathrm{N}-\mathrm{H} \bullet \bullet \mathrm{N}, \mathrm{NH} \bullet \bullet \mathrm{F}, \mathrm{C}-\mathrm{H} \bullet \bullet \mathrm{N}, \mathrm{C}-\mathrm{H} \bullet \bullet \mathrm{F}$ and $\mathrm{C}-\mathrm{H} \bullet \bullet \bullet \mathrm{n}$ hdrogen bonds and $\mathrm{F} \bullet \bullet \cdot F$ and $\Pi \bullet \bullet \bullet \Pi$ interactions. In ortho isomer, the molecular chains formed with $\mathrm{N}-\mathrm{H} \bullet \bullet \bullet \mathrm{N}$ hydrogen bonds are further interlinked with $\mathrm{C}-\mathrm{H} \bullet \bullet \bullet$ and $\mathrm{F} \bullet \bullet \mathrm{F}$ interactions. In the meta isomer, the $\mathrm{NH} 2$ groups from the four adjacent molecules form a cyclic arrangement leading to the formation of a rarely observed $\mathrm{N}-\mathrm{H} \bullet \bullet \mathrm{N}$ tetramer unit across the centre of inversion whereas, in case para, the packing is once again through the $\mathrm{N}-\mathrm{H} \bullet \bullet \mathrm{N}$ catemeric chains along with the $\mathrm{C}$ - $\mathrm{H}$...pi chains interconnected via $\mathrm{N}-\mathrm{H} \bullet \bullet \mathrm{F}$ interactions. The variety of interactions offered by these three isomers brings out the influence of weak interactions involving 'organic' fluorine in generating the packing motifs.

[1]Chopra,D et al.,(2006). Crystal growth \& design,6,843-845.

[2]Chopra,D et al.,(2007).J.Ind.Ins.Sci.,87,167-211.

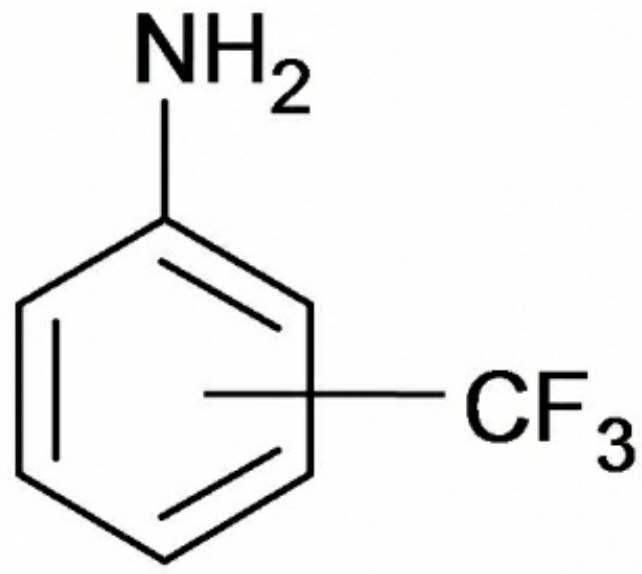

Keywords: In-situ crystallography, synthon, weak interactions 